QUADERNS DE FILOSOFIA VOL. VIII NÚM. 2 (2O2 I): IOI-I 2

eISSN: 234 I-3042 DOI: I 0.7203/QFIA. 8.2.2 I 380

María Ávila Bravo-Villasante

Universidad Rey Juan Carlos

\title{
La despolitización del feminismo en los discursos gerenciales
}

\author{
The depoliticization of feminism in managerial discourses
}

Recibido: 23/7/2021. Aceptado: 1/10/2021

Resumen: En su libro Mujeres y discursos gerenciales. Hacia la autogestión feminista (2020), María Medina-Vicent aborda desde una perspectiva crítica feminista los discursos gerenciales dirigidos a las mujeres, desvelando el androcentrismo y la presencia -y perpetuación - de tradicionales roles y estereotipos de género en los modelos de gestión. Mi propuesta pretende incidir en dos aspectos del análisis realizado por Medina-Vicent, por un lado, remarcar los peligros de la despolitización de los discursos gerenciales dirigidos a mujeres - sobre todo en tanto que la literatura gerencial acaba por informarnos a todas-. Por otro, profundizar en la crítica que la autora realiza de la cooptación del feminismo por parte de estos discursos.

\begin{abstract}
In her book Mujeres y discursos gerenciales. Hacia la autogestión feminista (2020), Medina-Vicent approaches management discourses aimed at women from a critical feminist perspective, revealing the androcentrism and the presence and perpetuation of traditional gender roles and stereotypes in management models. My proposal aims to highlight two aspects of Medina-Vicent's analysis: on the one hand, to highlight the dangers of depoliticizing management discourses aimed at women - especially insofar as management literature ends up informing us all. On the other hand, the author's critique of the co-optation of feminism by these discourses will be explored in greater depth.
\end{abstract}

Palabras clave: discursos gerenciales, teoría feminista, antifeminismo, despolitización.

Keywords: managerial discourses, feminist theory, antifeminism, depoliticization. 


\section{INTRODUCCIÓN}

$\mathrm{E}$ l libro de Maria Medina-Vicent, Mujeres y discursos gerenciales. Hacia la autogestión feministas, supone un análisis innovador de los discursos gerenciales dirigidos a mujeres. Una de las muchas virtualidades del trabajo, es analizar la influencia de estos discursos en la elaboración de la subjetividad de las mujeres. En Capital e ideología, Thomas Piketty insiste en remarcar que lo que mueve el mundo son las ideas. El asunto no es nuevo, ahora bien, si lo que mueve el mundo son las ideas, tenemos que ver qué es lo que mueve a estas. Es la tarea que emprende Medina-Vicent con su análisis, mostrarnos las narrativas claves propulsoras de estas ideas.

Medina-Vicent intenta dar respuesta a la cuestión que se interroga por la expansión e influencia de los discursos gerenciales. ¿Cómo es posible que discursos, a priori, destinados a un grupo reducido de mujeres, acaben convirtiéndose en referentes en los que mirarnos para la construcción de nuestra subjetividad? En su intento de resolver esta cuestión, la autora tiene que conjugar y encajar diversos marcos conceptuales (filosóficos, económicos y feministas). Esta mirada interdisciplinar a los discursos gerenciales hace, si cabe, mucho más valiosa su propuesta.

Una de las partes más interesante del análisis realizado por Medina-Vicent es poner de manifiesto la adaptación, acogida y trasmisión que los discursos gerenciales destinados a mujeres realizan de los grandes mantras neoliberales - la meritocracia, la capacidad de autogestión o la autoexigencia-. En este sentido, una de las primeras cuestiones a las que hay que hacer referencia es a los valores que el neoliberalismo pone en juego para que cada individuo se haga responsable de aquello que un sistema extremadamente competitivo considera triunfos, pero, sobre todo, de aquello que veladamente, permanece inaccesible para la mayoría. En este sentido, el discurso de la meritocracia nos lleva a obviar "todos los condicionantes materiales o simbólicos a los que hacen frente las mujeres" (MEDina-VICENT 2020, 46). Nos encontramos ante una estrategia profundamente reaccionaria con una larga trayectoria política: convertir los problemas sociales y estructurales (en especial los de las mujeres), en problemas personales. Lo personal deja de ser político y lo político se convierte en personal. La segunda parte de mi propuesta es analizar la cooptación del feminismo por parte de los discursos gerenciales dirigidos a mujeres. Si el feminismo es ante todo una teoría política, y los discursos gerenciales se presentan despolitizados ¿Qué feminismo es capaz de soportar una hibridación tal? Solo podría hacerse cargo un feminismo despolitizado. Siguiendo el análisis realizado por Sonia Reverter, un feminismo despolitizado es un feminismo al que le ha sido usurpada la 
agenda feminista en tanto que agenda política que busca transformar las sociedades (Reverter 2020). No es viable alcanzar la igualdad sin llevar a cabo cambios estructurales.

\section{EMPRENDE, GESTIONA, LIDERA IY CUIDA!}

Tal y como pone de manifiesto Medina-Vicent, los valores neoliberales están internamente ligados a la cuestión de la despolitización del discurso y a la creación de subjetividades capaces de dar respuesta a un sistema productivo marcadamente individualista. La autora muestra el vínculo que conecta la despolitización de los discursos gerenciales con la psicologización de la sociedad. Ante las actuales condiciones de precarización social nos encontramos como respuesta la austeridad política que testimonia la muerte más anunciada de la filosofía política: la del Estado de bienestar. Si se permite la metáfora, la actual crisis provocada por la pandemia de Covid-19 ha trasladado a los Estados benefactores a cuidados paliativos ${ }^{1}$. No solo debemos preguntarnos quién cuida de este agonizante espectro, además, es muy pertinente interrogarse por quién cuida lo que él ha dejado de cuidar. Ante la incapacidad de dar respuesta a la vulnerabilidad, se nos exhorta a una suerte de noción de resiliencia individual, como si esta siempre resultara un éxito (BUTLER; GAMBETTI y SabSAY 20I6).

Los discursos gerenciales estimulan esta noción de resiliencia individual, en la medida que asumen acríticamente la despolitización. Por un lado, son narrativas muy potentes, proferidas por mujeres referentes, que acaban informando a un amplio sector de la sociedad — más allá del reducido círculo de mujeres gerentes a los que se destinan estas propuestas-. Por otro lado, estos discursos acaban modelando subjetividades individuales acríticas con la dimensión estructural de la injusticia; desde este modelaje se asume que los conflictos socioeconómicos que atraviesan a los individuos no son asuntos políticos, sino resultados de una mala gestión personal. Por tanto, estamos ante otra vía de despolitización de las mujeres y, por ende, de la sociedad.

Otra de las cuestiones que, desde una perspectiva feminista, no podemos obviar es la adherencia acrítica de estas narrativas a lo que Medina-Vicent ha denominado cierto "esencialismo ambiguo" (MedinA-VICENT 2020, 40). Cuando la autora disecciona los discursos del liderazgo femenino, descubre toda una serie de lugares comunes sobre comunicación femenina y

1 Mientras esto sucede los niveles de pobreza se disparan. El Banco Mundial estima que los efectos de la pandemia de Covid-19, llevarán unos 150 millones de personas a la pobreza extrema a finales de 2021 (LAKNer; Nishant; Gerszon; Castaneda y Wu 202 I, January 11). 
masculina que parecen remitirnos al intergaláctico escenario propuesto por John Gray en su obra Los hombres son de Marte y las mujeres de Venus. Lejos de ser algo anecdótico, la relación entre discursos gerenciales, psicología pop y cierto "biologicismo posfeminista" (Favaro y De Miguel 20i6) resulta evidente. Como decíamos, de un modo acrítico en estos discursos se identifican valores "que muestran una tendencia concreta hacia un modelo de liderazgo más cercano al esencialismo y al estereotipo de género femenino tradicional: emoción, empatía, pensamiento relacional, capacidad multitarea, colaboración y comunicación inclusiva” (Medina-Vicent 2020, 42). Estamos, a todas luces, ante cualidades que deberían reconocerse no como femeninas o masculinas, estamos ante cualidades deseables para el conjunto de los seres humanos, y desde hace tiempo, se reclama que se eleven a la categoría de lo universal y dejen de formar parte de la otredad. Precisamente una de las primeras tareas que tuvo que abordar el feminismo — desde sus inicios ilustrados - fue rebatir la idea de una naturaleza diferente y complementaria de los sexos, reclamando, entre otros, la gran importancia de una educación en igualdad. Retomaremos estas cuestiones cuando analicemos la cuestión del work-life balance.

El segundo discurso que analiza Medina-Vicent es el del emprendimiento. Lo primero que llama nuestra atención, es la amplia acogida y difusión que ha alcanzado esta narrativa. ¿Cómo es posible que un discurso destinado a un perfil de mujer reducido tenga una recepción tan amplia y se convierta en un relato tan exportable? Aquí tenemos otra de las virtualidades del análisis de Medina-Vicent, nos descubre un aspecto nuclear del discurso neoliberal. Si el discurso sobre el emprendimiento tiene tanta aceptación entre las mujeres es por la facilidad con la que se puede extrapolar del plano empresarial, al laboral y personal. En cierta medida, este deslizamiento entre planos resulta eficaz en la medida en que se nos ha convertido en gestoras de nuestro propio negocio, y este negocio que parece ser el de todas, excede lo profesional. Por decirlo de un modo claro, tenemos en exclusiva la gestión de la esfera familiar — la familia es nuestro negocio, como también lo será la gestión que realicemos entre la esfera familiar y el resto de las esferas, en especial la profesional-. El discurso del emprendimiento, como señala Medina-Vicent, "se construye como una posibilidad para tomar las riendas de la propia vida y de su presencia en el ámbito laboral" (Medina-Vicent 2020, 43).

Es fácil percibir la coherencia que guardan estos discursos con las demandas planteadas por el sistema neoliberal a las identidades subjetivas. De este modo, la capacidad de autogestión se vuelve un requisito indispensable para el sistema de producción individualista que el sistema requiere, a saber, un sujeto responsable de modo absoluto de su vida, de sus circunstancias 
y de los contextos (que obviamente le exceden y superan). Este trasvase de responsabilidades desde el ámbito de la responsabilidad política al personal se blinda cuando lo hacen depender de la autonomía y de la libertad. En La sociedad del cansancio, Byung-Chul Han, nos habla de cómo el sujeto se positiviza "la transformación de sujeto en proyecto no hace que desaparezcan las coerciones. La coerción externa es reemplazada por una autocoerción que se hace pasar por libertad" (HAN 20 I 8, 96). De este modo, categorías como explotación u opresión dejan de tener sentido, como mucho estaríamos hablando de autoexplotación, y, a fin de cuentas, esta es siempre voluntaria (libre y autónoma). Con este ardid, la explotación, se transforma en una suerte de autoexplotación voluntaria, la adicción al entusiasmo de la que nos habla Remedios Zafra (2017). La estrategia no funcionaría sin un tercer elemento, al que ya hemos hecho alusión: la meritocracia. La autogestión y el entusiasmo son posibles allí donde nos encontramos abierta la posibilidad de logro. La "tiranía del mérito" (SANDEL 2020) se nutre de las teorías del esfuerzo, de la superación personal y de los discursos provenientes de la psicología pop que insisten en recordarnos que lo posible es, más allá de condicionantes materiales y sociales. Si todo esto no fuera suficiente, contamos con un sistema de valores que adecuadamente activados pueden reforzar la estrategia. Uno de los valores en alza es el de la ambición. La ambición, hasta ahora considerada deseable para los varones y perversa en las mujeres, se acaba transformando en un valor universal. Los valores asociados al cuidado no han tenido la misma suerte. $Y$ eso pese a que cada vez somos más conscientes, individual y colectivamente, de nuestra vulnerabilidad.

Una de las grandes apuestas del feminismo es, precisamente, convertir en una prioridad universalizar el cuidado como corresponsabilidad. María José Guerra considera que un "programa a discutir es el de desnaturalizar — no nos tiene que tocar en exclusiva a las mujeres - desprivatizar — considerarlo una responsabilidad pública-y desmercantilizar el cuidado" (Guerra 20I9). Del mismo modo la "ambición" se ha elevado a valor universal, sería deseable que también lo hicieran los valores asociados a la esfera de cuidado —empatía, sororidad/solidaridad, cooperación-. Sin embargo, en la literatura gerencial, y en especial, en los discursos gerenciales dirigidos a mujeres, este ascenso a lo universal está lejos de producirse, permanece vinculado de un modo acrítico a lo femenino. Lo que vemos es la asunción generalizada del cuidado como un asunto específicamente de las mujeres. La inclusión de la temática en la propia literatura, como señala Medina-Vicent, marca una diferencia sustancial con los discursos dirigidos a varones. No solo el cuidado corresponde a las mujeres, al igual que ocurre en sectores amplios de la sociedad, estas narrativas consideran que la conciliación personal, laboral y familiar es también problema suyo. 


\section{Discursos gerenciales para mujeres, Feminismo Y ARGUMENTOS REACCIONARIOS}

Hay una cuestión sobre la que es importante incidir. El individualismo inherente a estos discursos permite un acoplamiento desclasado, desracializado y deslocalizado, pese a que las autoras son conscientes de dirigirse a una élite. Sandberg reconoce que muchas partes de su obra son más pertinentes "para aquellas mujeres que son suficientemente afortunadas para poder elegir cuánto, cuándo y dónde desean trabajar, y otras partes podrán aplicarse a situaciones a las que se enfrentan las mujeres en cualquier contexto laboral, social y familiar" (SANDBERG 20I3, 10). Las autoras de estos discursos son mayoritariamente blancas, de clase alta, heterosexuales y occidentales - Ivanka Trump, Anne-Marie Slaughter o Sheryl Sandberg- Sin embargo, vemos que el número de mujeres interpeladas por estos mensajes excede a ese minoritario grupo de referencia. Esta cuestión es absolutamente relevante en tanto que nos permite dar cuenta de la despolitización absoluta del discurso: consigue desarticular dentro de su discurso los grandes movimientos políticos que se han mostrado capaces de ofrecer resistencias (y no resiliencia) al sistema: movimiento obrero, movimientos antirracistas, movimientos LGTBI y los movimientos poscoloniales. ¿Qué ocurre con el feminismo? Como remarca Medina-Vicent, se produce una cooptación de este por parte de estos discursos eliminando su carga política. Catherine Rottenberg (2013) manifestaba su preocupación por el desplazamiento del feminismo liberal por parte del feminismo neoliberal ¿es necesario un feminismo informado por las normas del neoliberalismo? Seguramente no, pero el análisis realizado de los discursos gerenciales dirigidos a mujeres pone de manifiesto que el neoliberalismo sí necesita de esta cooptación.

Mas allá de nuestras sospechas hacia lo que se ha dado en llamar "feminismo neoliberal" (Medina-Vicent 2018; Ávila 20 I9), intentaremos centrarnos en la hibridación que se produce entre discursos gerenciales y feminismo neoliberal. La cuestión que me gustaría plantear es la siguiente ¿̨El feminismo neoliberal inherente a ciertos discursos gerenciales puede llevar implícitos determinados sesgos sexistas que pueden desencadenar un efecto reactivo? Lo plantearé de otra forma ¿estamos ante discursos que aceptan acríticamente supuestos que acaban por asumir perspectivas antifeministas?

Uno de los aspectos en los que se hace evidente tanto la cooptación del feminismo por los discursos gerenciales dirigidos a mujeres como las contradicciones internas que esta asimilación produce es la cuestión del work-life balance. Aludíamos a que en este tipo de discursos se asume que el tema de gestión del cuidado es un tema que afecta a las mujeres (de ahí su inclusión en este tipo de literatura y no en la destinada a varones). Dado que estamos ante 
discursos que eliminan todo tipo de contextos social, político y económico y trasladan la responsabilidad a la capacidad gestora individual de las personas, el éxito y el fracaso de esta empresa (que no es ni más ni menos que el cuidado y mantenimiento de la vida) depende en exclusiva de cada mujer individual.

Hay voces que nos previenen de la imposibilidad de la contienda desde planteamientos extrańamente cercanos a los argumentos de la reacción contrafeminista y de carácter conservador que se produce en la década de los ochenta en Estados Unidos. Siguiendo los análisis de Susan Faludi (1983), la gran reacción de los 80 consiguió convencer a la opinión pública de que la culpa de todo la tiene el feminismo - la crisis económica, el aumento de violaciones el fracaso escolar adolescente, la pérdida de los valores, la infertilidad o el aumento de la delincuencia femenina-. La respuesta que nos da Anne-Marie Slaughter a la pregunta “¿pueden las mujeres tenerlo todo?” no está exenta de sesgos reaccionarios. Por un lado, se declara completamente comprometida con la igualdad de oportunidades entre mujeres y hombres. Sin embargo, dice renunciar a la "narrativa feminista" con la que creció. Lo que esta narrativa parecía sostener era que la batalla feminista se ganaría cuando tuviéramos reconocimiento, valoración y poder. Sin embargo, sostiene Slaughter, la igualdad real nunca se conseguirá mientras no se logre la otra mitad de la batalla: la de la familia. Ya tenemos enunciados los elementos que hay que poner en la balanza.

Para la autora, la igualdad real no sólo significa la valoración y el reconocimiento de las mujeres en los mismos términos que los hombres, significa la creación de una gama mucho más amplia. La igualdad real significa valorar la familia tanto como el trabajo y comprender que ambos ámbitos se refuerzan mutuamente. Esto requiere la incorporación de los hombres al mundo del cuidado, convertir la revolución feminista en una revolución humanista. La estrategia de despolitizar el feminismo no es nueva, como tan poco lo es hacer un feminismo sin feminismo, o intentar superar el feminismo con un humanismo - lo que supone, en cierto modo, reconocer que el feminismo no tiene sentido porque no tiene razón de ser- Recordemos que en el contexto de reacción conservadora y contrafeminista de los 80 Betty Friedan aboga por suavizar el feminismo radical y disfrazarlo de una suerte de política humanística desgenerizada (Friedan I 983). Obviando el tema del viraje al humanismo, la objeción que se le puede hacer a Slaughter es, precisamente, señalar al feminismo como responsable de la angustia que experimentan las mujeres profesionales.

El viejo mantra reaccionario "las mujeres no lo pueden tener todo" vuelve a escena y lo hace de la mano de una mujer que se autodesigna como feminista y posee poder, reconocimiento y valoración - fue Decana en la Princeton's Woodrow Wilson School of Public and International Affairs y Directora de Planificación de Políticas en el Departamento de Estado durante el 
gobierno de Obama- La revolución feminista requiere la incorporación de los hombres al mundo del cuidado, pero las interlocutoras a las que tiene en cuenta cuando habla sobre los motivos que la llevaron a abandonar su empleo son las mujeres. Así, en la charla TED (SLAUGHTER 20I3) que ofreció un año después a la publicación de su artículo, califica su decisión de abandonar su empleo para cuidar a sus hijos adolescentes como una decisión "responsable" y fruto del "amor". Si su decisión es responsable y fruto del amor, la decisión de quienes priorizan su carrera profesional sobre su vida privada ¿es irresponsable y fruto del egoísmo? ¿qué ocurre con el $99 \%$ de las personas que no pueden elegir? ¿Problemas estructurales como son la corresponsabilidad deben ser asumidos por cada mujer de manera individual? ¿Esta es la nueva narrativa feminista que propone Slaughter? ¿Qué narrativa feminista es la culpable de que los hombres no se incorporen al cuidado? Y por último ¿Es realmente necesaria la transformación de la revolución feminista en una revolución humanista? Slaughter parece estar más informada por las narrativas reaccionarias de los años 80 que por la narrativa feminista. Sus argumentos se dirigen a la "narrativa" equivocada: las narrativas feministas luchan porque las mujeres "puedan tenerlo todo", donde este todo no es otra cosa que la igualdad, donde ese "tenerlo todo" es tener lo mismo que pueden tener sus compañeros de viaje.

El error que comete Slaughter es bastante frecuente. Es un lugar común en la amalgama teórica posfeminista reducir la teoría feminista a una suerte de manual para alcanzar la cima en la sociedad neoliberal. Nada más lejos de la realidad. Si algo sabemos de teoría feminista es que no proporciona fórmulas individuales, como movimiento es colectivo, como teoría política es radical —en tanto que se dirige a la raíz de los problemas - y vinculada a la transformación social.

El dilema al que se enfrenta Slaughter, es el mismo al que se enfrentan muchas familias, la mayoría ajenas, e incluso contrarias, a las narrativas feministas a las que culpa Slaughter. En su discurso se obvia que solo desde una posición privilegiada — en la que se hacen evidentes los privilegios de clase, pero también, extrapolando la crítica que hooks le hace a Sandberg, privilegios raciales y heterosexuales - se puede elegir. Lo que podíamos esperar de una referente feminista es que trasladara el problema al ámbito de la responsabilidad política. Pero Slaughter, lo traslada al ámbito de la responsabilidad individual obviando que su decisión es una decisión que solo puede tomar quien efectivamente puede tenerlo todo. Supone que todo el mundo tiene capacidad — realde elección y puede tomar decisiones fruto del amor y de la responsabilidad. Porque lo que Slaughter parece decir es que es el egoísmo y la irresponsabilidad lo que lleva a las mujeres a apostar por su vida profesional — como si las facturas no las pagáramos con responsabilidad: precisamente la responsabilidad 
que nos lleva a acudir a nuestros puestos de trabajo todos los días-. Esto no es nuevo. Todo el argumentario reaccionario que muestra al feminismo como un movimiento egoísta y contra la familia hunde sus raíces en las primeras luchas contra el sufragismo y reaparece con cierta regularidad. Sería interesante ampliar la denuncia de la cooptación del feminismo por parte de los discursos gerenciales con un análisis de los discursos reaccionarios y ostensiblemente antifeministas que incorpora.

Volvemos a la pregunta ¿Pueden las mujeres tenerlo todo? De momento solo los hombres parecen poder tenerlo todo donde ese "todo", en este contexto, es una vida balanceada. El problema es que estas autoras omiten hacer la pregunta que todas estamos esperando ¿Por qué nosotras no podemos tenerlo todo - equilibrio entre vida personal, profesional y familiar - y ellos sí? La respuesta a esta pregunta es, inexcusablemente política y, por tanto, resulta incompatible con narrativas individualistas. Como ha puesto de manifiesto Medina-Vicent este tipo de discursos contribuyen a fundamentar estructuras de poder desiguales entre mujeres y hombres (Medina-Vicent 2020, 59).

Sheryl Sandberg, considera que "tenerlo todo" es la mayor trampa que se le ha puesto a las mujeres. "Pronunciadas en discursos e incluidas en titulares y artículos, estas dos palabras pretenden ser abanderadas de la ambición, pero en lugar de ello nos hacen sentir a todas que hemos fracasado" (SANDberg 2013,122). "Tenerlo todo" es una idea peligrosa, nadie puede tenerlo todo. Y, sin embargo, como pone de manifiesto bell hooks, el verdadero problema es que Sandberg lo tiene todo y no tiene ningún pudor en hacer ostentación de ello. Simboliza el "tipo de chica que lo tiene todo": blanca, rica y casada con un maravilloso marido al que, no debemos olvidar, dedica el libro "por hacer todo posible". hooks compara el modo de proceder de Sandberg con el de los vendedores de coches usados: empuja su producto, y lo empuja bien. Su lenguaje es $\tan$ bueno, $\tan$ lleno de contenido que ciertamente nos inclina a pasar por alto todo lo que no se dice (ноокs 20 13, 28 October).

El análisis que Medina-Vicent realiza sobre la cuestión del work-life balance nos permite retomar la cuestión que interroga sobre el feminismo al que invocan los discursos gerenciales, si bien, no ahonda en la falta de interseccionalidad de estos discursos. En este sentido, nos parece interesante incorporar la crítica que bell hooks realiza a la obra de Sandberg. En su artículo "Dig Deep: Beyond Lean In", hooks la acusa de presentar una descripción bastante simplista del feminismo. Sandberg desconoce las fuentes primarias, esto es, el trabajo teórico feminista. El feminismo que presenta recurre a categorías simples — hombres y mujeres-. Como sabemos las cosas no son tan sencillas. El trabajo teórico abordado por las diversas corrientes feministas — feminismos negros, de color, poscoloniales/decoloniales, los feminismos socialistas, femi- 
nismos lesbianos- insiste en abordar y comprender la manera en que la raza, la clase y la sexualidad interseccionan con el género.

La crítica que hooks le hace a Sandberg podemos hacerla extensible al resto de discurso gerenciales analizados por Medina-Vicent. Según la versión simplista del feminismo que manejan estos discursos, este quedaría reducido a la consecución de la igualdad de género dentro del sistema social existente, por lo que las estructuras patriarcales capitalistas quedarían intactas, esto es, seguiríamos en un sistema profundamente imperialista, racializado, burgués y heteronormativo.

\section{Conclusiones}

En la medida en que los discursos gerenciales dirigidos a mujeres son un espejo de la sociedad, no podemos omitir realizar un análisis crítico de los mismos. Sin duda alguna, la cuestión que interroga por el feminismo invocado por estos discursos sigue sin tener respuesta fácil. El feminismo al que aluden estos discursos se parece más al postfeminismo en su versión popular, donde el feminismo remite a un imaginario de mujeres poderosas, seguras de sí mismas y libres de elegir. Un posfeminismo que asume acríticamente el mito de la libre elección (De Miguel 20 I 5) y nos lleva a vivir en un espejismo según el cual somos libres para elegir todo: elegimos a nuestro socio de vida (SANDBERG 2013), la imagen que proyectamos, la gestión de nuestras vidas (y sus múltiples esferas), lo que compramos, lo que vendemos. Elegimos el momento y el lugar adecuado - llegado el caso elegimos cuántos óvulos congelar, cuántos fecundar-. Elegimos triunfar o no hacerlo — recordemos que Sandberg sospecha que si las mujeres no triunfamos es porque tenemos miedo a triunfar-. Estamos ante un imaginario extremadamente individualista en el que no queda rastro de la política feminista en tanto agenda política capaz de transformar el mundo (REVERTER 2020).

El análisis crítico elaborado por Maria Medina-Vicent nos ha permitido analizar la despolitización de los discursos gerenciales destinados a mujeres, despolitización no ajena a los requisitos de un sistema individualista de producción que requieres sujetos atomizados. Una de las tareas pendientes, haciendo extensible la crítica de hooks a Sandberg es ampliar la crítica feminista a estos discursos analizando el subtexto de clase, raza y sexualidad. 


\section{REFERENCIAS BIBLIOGRÁFICAS}

Ávila Bravo-Villasante, M. 20 I9, La máquina reaccionaria. La lucha declarada a los feminismos, Valencia: Tirant Humanidades

Butler, J.; Gambetti, Z. y Sabsay, L. 20i6, "Introduction”, J. Butler, Z. GamBetTi y L. Sabsay (ed.), Vulnerability in resistance, Durham y Londres: Duke University Press.

De Miguel, A. 20 i 5, Neoliberalismo sexual. El mito de la libre elección, Madrid: Cátedra.

FALUdi, S. I993, Reacción. La guerra no declarada contra la mujer moderna, Barcelona: Círculo de lectores.

Favaro, L. G. y De Miguel, A. 20i6, “¿Pornografía feminista, pornografía antirracista y pornografía antiglobalización? Para una crítica del proceso de pornificación cultural", Labrys, Études Féministes/Estudos Feministas, 29.

Friedan, B. I983, La segunda fase, Barcelona: Plaza y Janes.

Han, B.-CH. 201 8, La sociedad del cansancio, Barcelona: Herder.

ноокs, bell (October 28, 20 I3), "Dig Deep: Beyond Lean In", The Feminist Wire, Recuperado de: http://thefeministwire.com/2013/10/17973/

Lakner, Ch.; Nishant, Y.; Gerszon Mahlerr, D.; Castaneda Aguilar, A. y Wu, H. (January 11, 202 I), "Updated estimates of the impact of COVID-19 on global poverty: Looking back at 2020 and the outlook for 2021", The World Bank Group. https://blogs.worldbank.org/opendatalupdated-estimates-impact-covid-19-global-poverty-looking-back-2020-and-outlook-2021

Medina-Vicent, M. 20i8, "Feminisme neoliberal: un oxímoron?", Quaderns de filosofía, V (2): 75-101. doi: 10.7203/qfia.5.2.13187

Medina-Vicent, M. 2020, Mujeres y discursos gerenciales. Hacia la autogestión feminista, Granada: Comares.

Piketty, Th. 20i9, Capital e ideología, Barcelona: Deusto.

Reverter Bañón, S. 2020, “¿Está reventando el capitalismo neoliberal la liberación de las mujeres?”, La Torre de Lucca, 9 (17), julio-diciembre: 193-201.

Rottenberg, C, 2013, "The Rise of Neoliberal Feminism", Cultural Studies, DOI: 10.1080/09502386.2013.857361. Recuperado de: http://www.bgu. ac.il/ $\sim$ rottenbe/The $\% 20$ rise $\% 20$ of $\% 20$ neoliberal\%20feminism.pdf

SAndberg, S. 2013, Lean In. Women, Work and the Will to Lead, Nueva York: Alfred A. Knopf.

SANDEL, M. J. 2020, La tiranía del mérito: ¿Qué ha sido del bien común?, Barcelona: Debate.

Slaughter, A.-M. 2013, “¿Podemos todos 'tenerlo todo'?”, TEDGlobal. Recuperado de: https://www.ted.com/talks/anne_marie_slaughter_can_we_all_ have_it_all/up-next?language=es 
Slaughter, A.-M. (July/August 20I2), "Why women still can't have it all", The Atlantic. Recuperado de: https://www.theatlantic.com/magazine/archive/2012/07/why-women-still-cant-have-it-all/309020/

Zafra, R. 20I7, El entusiasmo. Precariedad y trabajo creativo en la era digital, Barcelona: Anagrama. 